\title{
Metodología para el Registro de Parámetros de Calidad de Energía en Microrredes Inteligentes
}

\section{Methodology for Registration of Power Quality Parameters in Smart Microgrids}

\author{
L.E Perdomo Orjuela ${ }^{1}$, A.A Rodríguez ${ }^{2}$, F Santamaría ${ }^{3}$ \\ ${ }^{1}$ Universidad Distrital Francisco José de Caldas,Colombia leperdomoo@correo.udistrital.edu.co \\ ${ }^{2}$ Universidad Distrital Francisco José de Caldas,Colombia aalfonsor@ correo.udistrital.edu.co \\ ${ }^{3}$ Universidad Distrital Francisco José de Caldas,Colombia fsantamariap@udistrital.edu.co
}

Recibido: mar 05, 2016. Aceptado: jun 12, 2016. Versión final: jun 16, 2016

\begin{abstract}
RESUMEN
El registro de parámetros de calidad de energía en todos los niveles de tensión de los sistemas eléctricos de potencia al igual que en microrredes inteligentes es una necesidad, por lo tanto, es necesario disponer de dispositivos que permiten la adquisición, el procesamiento y el análisis de estos parámetros. En este artículo se presenta una metodología para la adquisición y procesamiento de la información, las estrategias de telecomunicaciones utilizadas y los criterios de compatibilidad electromagnética para el diseño del circuito impreso. Esta metodología permite obtener un dispositivo con alta fiabilidad y bajo costo, en términos de producción y operación. Además, al tener múltiples registradores conectados a una red de tensión inferior a $1 \mathrm{kV}$, es posible inferir algunas características del origen de los disturbios registrados.
\end{abstract}

Palabras Clave: Microrred, Internet de las cosas, Compatibilidad Electromagnética, Circuito impreso, calidad de potencia

\begin{abstract}
Registration of power quality parameters on smart microgrids is a need, therefore, it is necessary to have devices that allow the acquisition, processing and analysis of these parameters. This article presents a methodology for the acquisition and processing of information, involving telecommunications strategies and electromagnetic compatibility criteria for designing printed circuit boards. This methodology allows to get a device with high-reliability and low cost, in terms of production and operation. In addition, by having multiple recorders connected to a network of less than 1 $\mathrm{kV}$ it is possible to infer some characteristics about the origin of the registered disturbances.
\end{abstract}

Keywords: Microgrid, Internet of Thinks, Electromagnetic Compatibility, Printed Circuit Board, Power Quality.

\section{INTRODUCCIÓN}

Actualmente, la inclusión de generación distribuida en niveles de media y baja tensión del sistema eléctrico interconectado, sumado a sistemas de almacenamiento local, medición de parámetros eléctricos y posterior transferencia mediante múltiples tecnologías de comunicación (telemetría), han permitido el abastecimiento local de cargas, asociado al concepto de administración de la energía, mediante desarrollos enfocados hacia el Internet de las Cosas - IoT [1].

Este tipo de redes, se encuentran en la capacidad de operar en modo isla, ante condiciones anormales presentadas en el sistema interconectado (inestabilidad en frecuencia y tensión), con la finalidad de minimizar los cortes de energía debidos a deslastres de carga, entre otros. En caso de operación de forma interconectada, los excesos de energía producto de la generación distribuida presente en la microrred, pueden ser entregados al sistema. Lo anterior se conoce como microrredes eléctricas autosuficientes e interconectadas a la red [1]-[4].

Este nuevo concepto de operación, control y administración del sistema, permite el incremento en confiabilidad del suministro de energía y mayor flexibilidad de operación del sistema, sin embargo, esto trae consigo nuevos retos en la medición de los parámetros de estado estable y calidad de energía [5][11]. 
Los fenómenos de calidad de potencia presentes en las microrredes eléctricas autosuficientes e interconectadas a la red, pueden deberse a múltiples factores [12]-[14]. El primero de ellos, referente a la circulación de armónicos de corriente y tensión, son producto de la operación de transformadores en zona de saturación, cargas electrónicas conectadas a la red (Inversores asociados a generación distribuida, computadores, dispositivos IoT), entre otros [2], [4], [15], [16]. Continuado con la descripción de los fenómenos, las sobretensiones transitorias de frente lento y rápido, pueden deberse a operaciones de maniobra en el sistema, impactos directos e indirectos de descargas eléctricas atmosféricas en las redes de media tensión - MV y posterior transferencia a las redes de baja tensión - LV, a través del acoplamiento capacitivo existente en los devanados del transformador de distribución [17]-[25]. Por último, la presencia de huecos de tensión en las microrredes, se asocia al proceso de desconexión por parte de una fuerte carga o central de generación del sistema interconectado, debido a deslastre de carga u operación de modo isla [26].

En vista de lo anterior, se ha decidido realizar una investigación asociada al desarrollo de prototipos electrónicos modulares y escalables, que permitan el adecuado registro de parámetros de estado estable y de calidad energía presentes en redes de tensión menor a 1 $\mathrm{kV}$. El presente artículo en la sección II describe la metodología para el registro de parámetros de calidad de energía presentes en microrredes eléctricas. La sección III da a conocer los resultados obtenidos en los diseños de tarjetas de circuitos impresos - PCB, empleando criterios de compatibilidad electromagnética - EMC, para la mitigación de interferencias internas y/o externas que perturben el registro y procesamiento de las señales adquiridas.

\section{METODOLOGÍA}

El desarrollo de un prototipo para el registro de parámetros de calidad de energía tiene múltiples desafíos entre ellos la diferencia de magnitud, frecuencia y periodicidad de las señales requeridas para evaluar los indicadores establecidos para cada parámetro. Por tal motivo se desarrolló la metodología de adquisición y procesamiento de información expuesta en la Figura 1.

La metodología propuesta tiene tres ejes fundamentales: la adquisición, el procesamiento y la trasmisión de la información. Cada uno de estos ejes tiene una diversidad de criterios que debieron ser identificados y acotados.

\subsection{Adquisición de información}

Los fenómenos de calidad de energía se diferencian entre otras cosas por la naturaleza de la señal analizada, la frecuencia de las señales, la magnitud y la periodicidad. Por tal motivo, como estrategia se identificaron las señales compartidas, los requerimientos de magnitud y frecuencia.

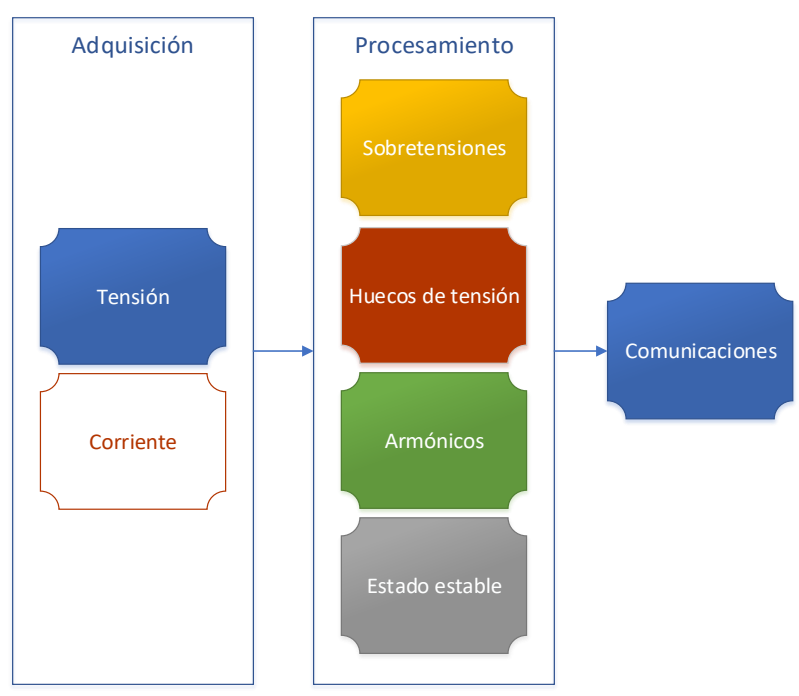

Figura 1. Metodología de adquisición y procesamiento. Fuente. Elaboración propia.

Una vez se identifica las señales que se comparten y sus características, se selecciona los sistemas de acondicionamiento y sus elementos constitutivos. Seguidamente se verifica los elementos para que cumplan con los requerimientos de magnitud y frecuencia en los rangos requeridos por los parámetros que las comparten.

A modo de ejemplo para seleccionar el acondicionamiento de tensión para estado estable, armónicos y sobretensiones se elige un divisor capacitivo, se calcula para la magnitud requerida en estado estable y en sobretensiones y se seleccionan capacitores para la magnitud máxima soportada y el rango de frecuencia tanto de estado estable como de transitorios.

\subsection{Procesamiento de información}

Debido a la frecuencia de las señales, la no periodicidad de algunas y los requerimientos diferentes de información, se debe utilizar un procesador diferente para cada uno de los parámetros registrados. Esto debido a que si se utilizara un solo procesador para la realización de tareas en paralelo, es necesario implementar un programador de tareas.

El problema del programador de tareas es el esquema de prioridad de tareas, ya que, al tener señales muy rápidas y no periódicas, se debe dedicar el procesador de manera exclusiva a esta tarea, perdiendo información valiosa de los demás fenómenos. 
Adicionalmente, hay señales que requieren una alta demanda de procesamiento, como trasformadas de Fourier o el manejo de un alto volumen de información producto de altas velocidades de muestreo.

Por tal motivo, es necesario disminuir la cantidad de datos digitalizados. Esto se logra, implementando un sistema de rangos, en el cual, se registra el momento en que la señal se encuentra dentro del rango y no el valor exacto de la misma.

\subsection{Transmisión de información}

El costo de trasmisión de datos a través de sistemas de comunicación está directamente relacionado con la cantidad de datos y la velocidad de transmisión. Por tal motivo, no se transmite las señales digitalizadas sino los datos procesados de cada indicador de los parámetros de calidad.

Adicionalmente, los datos son enviados hacia el servidor con una periodicidad definida entre envío y evitando enviar datos en cero, lo que permite disminuir la cantidad de datos enviados y la velocidad requerida de trasmisión. Dentro del desarrollo del esquema de comunicaciones es necesario tener en cuenta que en muchos lugares del mundo no se cuenta con red celular $3 \mathrm{G}$ o conexión a internet. Por esta razón, se debe incluir un sistema para trasmisión de datos fuera de línea como lo muestra la Figura 2.

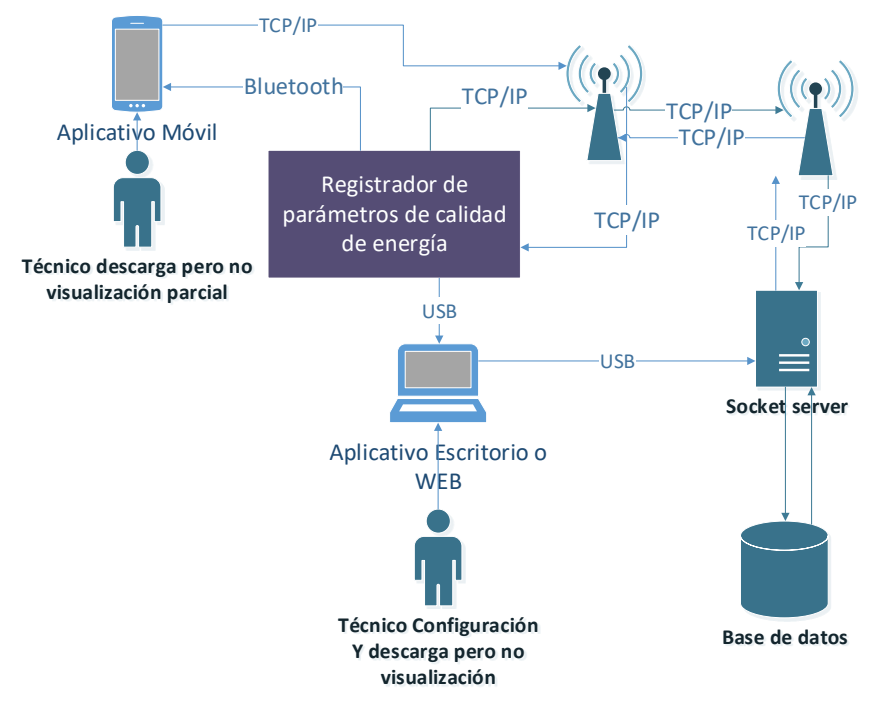

Figura 2. Esquema de comunicaciones. Fuente. Elaboración propia.

El sistema de trasmisión de datos fuera de línea requiere almacenar los datos de manera local y proveer de un sistema de comunicaciones que permita a un usuario realizar la descarga de manera local y trasportar los datos hasta un lugar donde se pueda realizar la conexión al servidor.

Esta estrategia de comunicaciones provee confiabilidad y seguridad al sistema, ya que se salvaguarda a toda costa los valiosos registros.

\section{RESULTADOS}

Los resultados a continuación presentados, corresponden a la determinación de la clase del prototipo, los rangos obtenidos para el registro de componentes armónicas de corriente y tensión, sobretensiones transitorias de frente lento y rápido y huecos de tensión, basado en la metodología descrita en la sección II. Posterior a la presentación de estos valores, se presenta la primera tarjeta de circuito impreso - PCB enfocada al registro de sobretensiones transitorias de frente lento y rápido presentes en redes de tensión menor a $1 \mathrm{kV}$.

\subsection{Determinación de la clase del prototipo}

En [27], se presenta tres tipos de clases (A, S y B) para la medición. A continuación, se presenta la descripción de cada clase:

- La clase A es utilizada en lugares donde es "necesaria una medición precisa".

- La clase $\mathrm{S}$ es adecuada para "aplicaciones estáticas tales como mediciones de calidad de energía”. Esta es similar a la clase A, pero con requerimientos más bajos.

El rango de medición para clase $\mathrm{S}$ es 42.5 - 69 $\mathrm{Hz}$, mientras que para clase A es $57.5-69 \mathrm{~Hz}$. En clase A la incertidumbre de medición no debe exceder $(+/-10 \mathrm{mHz})$ y para la clase $\mathrm{S}$ no debe exceder $(+/-50 \mathrm{mHz})$. Para valores de tensión de alimentación obtenidos mediante una relación de transformación $\mathrm{U}_{\mathrm{din}}$, en los rangos de $10 \%-150 \% \mathrm{U}_{\mathrm{din}}$, el valor de incertidumbre en la medición para la clase $\mathrm{A}$, no debe excerder +/- $0.1 \%$ de $\mathrm{U}_{\text {din }}$, contrastando con el valor de la Clase $\mathrm{S}$ en la cual el valor no debe exceder $+/-$ $0.5 \%$ de $\mathrm{U}_{\text {din. }}$. Otro tipo de requerimientos pueden ser el valor de tensión $\mathrm{U}_{\mathrm{rms}}$, swell, Dip, interrupciones y desbalances de tensión.

- La clase B hace referencia a evitar la fabricación de instrumentación obsoleta existente. Se hace la salvedad de no aplicar a desarrollos nuevos.

Se decidió adoptar para el desarrollo adecuado del proyecto, un módulo de medición de armónicos de corriente y tensión clase A. 
3.2 Determinación de las características principales a registrar de los armónicos de tensión y corriente

De acuerdo a [28], los límites de valores de distorsión armónica de tensión clasificados en rangos de tensión son:

- $1 \mathrm{kV}-69 \mathrm{kV}: D_{V}=3 \%, T H D=5 \%$

- $69 \mathrm{kV}-161 \mathrm{kV}: D_{V}=1.5 \%, T H D=2.5 \%$

- Mayor a $161 \mathrm{kV}: D_{V}=1 \%, T H D=1.5 \%$

La distorsión armónica individual Dv, hace referencia a la razón de la magnitud de la componente armónica individual respecto a la magnitud de la componente fundamental, expresada en un valor porcentual. La distorsión armónica total de tensión THDv hace referencia a la razón entre la raíz cuadrada de la suma de todos los cuadrados de la magnitud de cada componente armónica respecto de la componente fundamental, expresada en un valor porcentual.

Según [28], los valores de distorsión armónica individual Di y la distorsión total de corriente THDi, se pueden determinar siguiendo un procedimiento similar a las de tensión, haciendo la salvedad que este procedimiento toma en cuenta las componentes armónicas de corriente. El valor distorsión total de la demanda - TDD, es proporcional al THDi, al multiplicar este último por un factor $\mathrm{k}$, el cual es la relación entre la magnitud de la componente armónica fundamental y la corriente de carga de demanda máxima en el punto de conexión común - PCC.

Los valores límite de distorsión de corriente armónica individual y de distorsión total de demanda, se toman según los presentado en la Tabla $\mathrm{V}$ de [28]. Para el rango de tensión $120 \mathrm{~V}-69 \mathrm{kV}$ se tiene los siguientes valores de TDD:

$$
\text { - } \frac{\mathrm{I}_{\mathrm{sc}}}{\mathrm{I}_{\mathrm{l}}} \leq 20 T D D=5 \%
$$$$
\text { - } \frac{\mathrm{I}_{\mathrm{sc}}}{\mathrm{I}_{\mathrm{l}}}=20 T D D<50 T D D=8 \%
$$$$
\text { - } \frac{\mathrm{I}_{\mathrm{sc}}}{\mathrm{I}_{\mathrm{l}}}=50 T D D<100 T D D=12 \%
$$$$
\text { - } \frac{\mathrm{I}_{\mathrm{sc}}}{\mathrm{I}_{\mathrm{l}}}=100 T D D<1000 T D D=15 \%
$$

- $\frac{\mathrm{I}_{\mathrm{sc}}}{\mathrm{I}_{\mathrm{l}}} \geq 1000 T D D=20 \%$

- Donde Isc es "la mínima corriente de cortocircuito trifásica" e Il es "la corriente de demanda máxima en el PCC" [28].

Acatando la sugerencia presentada en [27], el módulo para el registro de armónicos de corriente y tensión, debe registrar hasta el cincuentavo 50th armónico respecto de la fundamental.

\subsection{Determinación de rangos para la clasificación de sobretensiones transitorias de frente lento y rápido}

De acuerdo a [29] y un estudio realizado por los autores [30], los valores tomados en cuenta para la clasificación de rangos de sobretensión en redes de tensión menor a 1 $\mathrm{kV}$ deben ser $500 \mathrm{~V}, 1000 \mathrm{~V}$ y $1500 \mathrm{~V}$. Por otro lado, con el fin de dar escalabilidad al prototipo, se ha dotado al mismo con la flexibilidad en la selección de los rangos por parte del usuario final.

\subsection{Clasificación de parámetos característicos de los huecos de tensión}

Según [31], la magnitud típica de la caída de tensión se encuentra en los valores de $0.9-0.1$ p.u. Por otro lado, este evento puede ser clasificado según su duración siendo esta instantánea, momentánea o temporal, cuyos valores corresponden a $0.5-30$ ciclos, 30 ciclos $-3 \mathrm{~s}$ y $3 \mathrm{~s}-1 \mathrm{~min}$.

Por tanto, se hace necesario clasificar el evento según su duración, asociado a la etiqueta de la magnitud de tensión en p.u.

\subsection{Clasificación de parámetos característicos de los huecos de tensión}

El prototipo para el registro de sobretensiones transitorias de frente lento y rápido, se presenta en la Figura 3.

Con el fin de realizar en adecuado registro y digitalización de las señales incidentes, se han implementado criterios de compatibilidad electromagnética - EMC [32]-[50], los cuales permiten mitigar perturbaciones de tipo conducido y radiado.

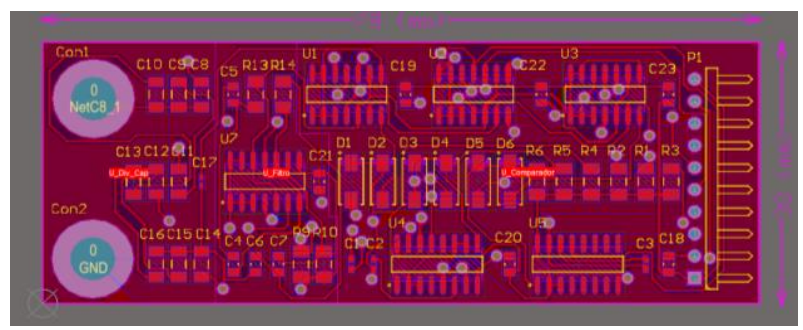

Figura 3. Módulo para el registro de sobretensiones transitorias de frente lento y rápido en redes de tensión menor a $1 \mathrm{kV}$. Fuente. Elaboración propia.

Las interferencias producto de acoplamientos galvánicos, son mitigadas mediante la incorporación capacitores de 1 $\mathrm{nF}$ en las proximidades de los circuitos integrados - CI, con el fin de minimizar los lazos inductivos. Sumado a lo anterior, el prototipo incorpora otra serie de medidas como son filtros de red, con el fin de eliminar rudo en modo común y diferencial, dimensionamiento del ancho y espaciamiento de las microcintas, incorporación del 
plano de tierra y distribución de los componentes de acuerdo a su frecuencia de operación y propósito.

El encerramiento del prototipo electrónico, ha sido diseñado para atenuar microondas en un espectro de frecuencia de hasta $10 \mathrm{MHz}$, con valores superiores a 100 dB.

\section{CONCLUSIONES}

Los valores típicos de sobretensiones transitorias en redes de tensión menor a $1 \mathrm{kV}$ son $500 \mathrm{~V}, 1000 \mathrm{~V}$ y 1500 . Las magnitudes esperadas respecto a las caídas de huecos de tensión se encuentran en el rango $0.9-0.1$ p.u. con duraciones entre $0.7 \mathrm{~ms}-1 \mathrm{~min}$, por lo tanto se requiere de sistemas de registro que permitan la adquisición de estos datos, con el fin de caracterizar y clasificar las perturbaciones presentes en microrredes de baja tensión.

El desarrollo de un prototipo para el registro de parámetros de calidad de energía tiene múltiples desafíos entre ellos la diferencia de magnitud, frecuencia y periodicidad de las señales requeridas para evaluar los indicadores establecidos para cada parámetro.

\section{AGRADECIMIENTOS}

Esta investigación es financiada por el Fondo Nacional para la financiación de la ciencia, la tecnología y la innovación "Fondo Francisco José de Caldas" del Departamento Administrativo de Ciencia, Tecnología e innovación - COLCIENCIAS (Contract: FP44842 - 321 2015).

\section{REFERENCIAS}

[1] C. Trujillo, F. Santamaria, J. Hernández, A. Jaramillo, E. Gaona, E. Rivas, O. Flórez, D. Rodríguez, J. Alarcón, and H. Rojas, Microrredes eléctricas, Primera. Bogotá: Universidad Distrital Francisco José de Caldas, 2016.

[2] H. Minxiao, S. Xiaoling, L. Shaobo, and Z. Zhengkui, "Transient analysis and control for microgrid stability controller," in 2013 IEEE Grenoble Conference, 2013, pp. 1-6.

[3] H.-L. Hsiang-Lin Huang, Y.-D. Yih-Der Lee, and Y.-R. Yung-Ruei Chang, "Improvement of transient response to islanding control of microgrid with bidirectional inverter," in 2015 International Conference on Renewable Energy Research and Applications (ICRERA), 2015, pp. 184-188.

[4] W. Wei Deng, W. Wei Pei, and Z. Zhiping Qi, "Impact and improvement of Distributed Generation on voltage quality in Micro-grid," in 2008 Third International Conference on Electric Utility Deregulation and Restructuring and Power Technologies, 2008, pp. 1737-1741.

[5] M. Begovic, Ed., Electrical Transmission Systems and Smart Grids: Selected Entries from the Encyclopedia of Sustainability Science and Technology. New York: Springer Science \& Business Media, 2012.

[6] F. Bouhafs, M. Mackay, and M. Merabti, Communication Challenges and Solutions in the Smart Grid. New York: Springer, 2014.

[7] Z. M. Fadlullah and N. Kato, Evolution of Smart Grids, vol. 13. New York: Springer Science \& Business Media, 2015.

[8] B. M. Buchholz and Z. Styczynski, Smart Grids - Fundamentals and Technologies in Electricity Networks. New York: Springer Science \& Business Media, 2014.

[9] T. Jiang, L. Yu, and Y. Cao, Energy Management of Internet Data Centers in Smart Grid. New York: Springer, 2015.

[10] K. C. Budka, J. G. Deshpande, and M. Thottan, Communication Networks for Smart Grids: Making Smart Grid Real. New York: Springer Science \& Business Media, 2014.

[11] A. Keyhani and M. Marwali, Eds., Smart Power Grids 2011. New York: Springer Science \& Business Media, 2012.

[12] CIGRE WG 37.28, Quality of Supply Customers Requirements, no. June. Folsom: International Council on Large Electric Systems, 2001.

[13] CIGRE WG C4.07, Power Quality Indices and Objectives, no. January. Folsom: International Council on Large Electric Systems, 2005.

[14] CIGRE JWG C4.107, Economic Framework for Voltage Quality, no. June. Folsom: International Council on Large Electric Systems, 2011.

[15] M. Hamzeh, H. Karimi, and H. Mokhtari, "Harmonic and Negative-Sequence Current Control in an Islanded Multi-Bus MV Microgrid," IEEE Trans. Smart Grid, vol. 5, no. 1, pp. 167-176, Jan. 2014.

[16] G. W. Chang, H. J. Su, L. Y. Hsu, H. J. Lu, Y. R. Chang, Y. D. Lee, and C. C. Wu, "A study of passive harmonic filter planning for an AC microgrid," in 2015 
IEEE Power \& Energy Society General Meeting, 2015, pp. 1-4.

[17] CIGRE WG C4.301, Use of Surge Arresters for Lightning Protection of Transmission Lines, no. 253. Folsom: International Council on Large Electric Systems, 2010.

[18] CIGRE WG01 SC33, Guide to procedures for estimating the lightning performance of transmission lines, vol. 01, no. October. Folsom: International Council on Large Electric Systems, 1991.

[19] CIGRE TF 33.01.03, Lightning Exposure of Structures and Interception Efficiency of Air Terminals, no. October. Folsom: International Council on Large Electric Systems, 1997.

[20] CIGRE WG 33.01.02, Characterization of Lightning for Applications in Electric Power Systems, no. December. Folsom: International Council on Large Electric Systems, 2000.

[21] CIGRE WG C4.4.02, Protection of MV and LV networks against lightning. Part I: Common Topics, no. 438 pt 1/2. Folsom: International Council on Large Electric Systems, 1997.

[22] CIGRE WG C4.404, Cloud to gound lightning parameters derived from lightning location systems, no. April. Folsom: International Council on Large Electric Systems, 2009.

[23] CIGRE WG C.4.402, Protection of Medium Voltage and Low Voltage Networks Against Lightning Part 2: Lightning protection of Medium Voltage Networks, no. December. Folsom: International Council on Large Electric Systems, 2010.

[24] CIGRE WG C4.408, Lightning Protection of Low Voltages Networks, no. August. Folsom: International Council on Large Electric Systems, 2013.

[25] CIGRE TF 33.01.02, Lightning Characteristics Relevant for Electrical Engineering: Assement of Sensing, Recording and Mapping Requirements in the Light of Present Technological Advancements. Folsom: International Council on Large Electric Systems, 1995.

[26] Z. Zhili Lei, X. Xin Ai, and M. Mingyong Cui, "Coordinated control strategy based on network parameters for voltage sags compensating in Microgrid," in 2010 5th International Conference on Critical Infrastructure (CRIS), 2010, pp. 1-5.

[27] IEC, IEC 61000-4-30 Electromagnetic compatibility (EMC) - Part 4-30: Testing and measurement techniques - Power quality measurement methods. Geneva: International Electrotechnical Commission, 2000.

[28] IEEE Power Engineering Society, IEEE Recommended Practices and Requirements for Harmonic Control in Electrical Power Systems, IEEE Std 519 - 1992, vol. 1992, no. June. New York: Institute of Electrical and Electronics Engineers, 1992.

[29] R. Transit, V. Interface, S. Committee, I. Vehicular, and T. Society, IEEE Recommended practice on Surge Voltages in Low-Voltage AC Power Circuits, IEEE C62.41.2-1991, vol. 2004, no. February. New York, 2005.

[30] A. Alfonso, L. Perdomo, F. Santamaria, and G. Carlos, "Transient surges analysis in low voltage networks," Rev. Tecnura, vol. 18, no. Edición especial doctorado, pp. 41-50, 2014.

[31] IEEE Power \& Energy Society, IEEE Std 1159 - IEEE Recommended Practice for Monitoring Electric Power Quality., vol. 2009, no. June. New York: Institute of Electrical and Electronics Engineers, 2009.

[32] J. Balcells, F. Daura, R. Esparza, and R. Pallás, Interferencias electromagnéticas en sistemas electrónicos. Ciudad de México: Alfaomega S.A, 1992.

[33] C. Bowick, RF Circuit Design. Indianapolis: Newnes, 1982.

[34] S. Celozzi, R. Araneo, and G. Lovat, Electromagnetic shielding. New Jersey: John Wiley \& Sons, 2008.

[35] M. Davidovitz, "Calculation of multiconductor microstrip line capacitances using the semidiscrete finite element method," IEEE Microw. Guid. Wave Lett., vol. 1, no. 1, pp. 5-7, Jan. 1991.

[36] DelingerEdgar, "A Frequency Dependent Solution for Microstrip Transmission Lines," IEEE Trans. Microw. Theory Tech., vol. 19, pp. 30-39, 1971.

[37] E. J. Denlinger, "A Frequency Dependent Solution for Microstrip Transmission Lines," IEEE Trans. Microw. Theory Tech., vol. 19, no. 1, pp. 30-39, Jan. 1971.

[38] K.-H. Gonschorek and R. Vick, Electromagnetic Compatibility for Device Design and System Integration. Heidelberg: Springer Science \& Business Media, 2009. 
[39] T. Laurila, V. Vuorinen, M. Paulasto-Kröckel, M. Turunen, T. T. Mattila, and J. Kivilahti, Interfacial Compatibility in Microelectronics: Moving Away from the Trial and Error Approach. London: Springer Science \& Business Media, 2012.

[40] R. Ludwig and G. Bogdanov, RF Circuit Design: Theory and Applications, London. New York: Prentice Hall, 2009.

[41] R. Mittra and T. Itoh, "A New Technique for the Analysis of the Dispersion Characteristics of Microstrip Lines," IEEE Trans. Microw. Theory Tech., vol. 19, no. 1, pp. 47-56, Jan. 1971.

[42] M. I. Montrose, EMC and the Printed Circuit Board: Design, Theory, and Layout Made Simple. New York: John Wiley \& Sons, 2004.

[43] M. I. Montrose, Printed Circuit Board Design Techniques for EMC Compliance: A Handbook for Designers, Second edi. New York: John Wiley \& Sons, 2000.

[44] D. Morgan, A Handbook for EMC Testing and Measurement. London: The Institution of Engineering and Technology, 1994.

[45] A. M. Niknejad, Electromagnetics for HighSpeed Analog and Digital Communication Circuits. Cambridge: Cambridge University Press, 2007.

[46] M. O'Hara, EMC at Component and PCB Level. Oxford: Newnes, 1998.

[47] H. W. Ott, Electromagnetic Compatibility Engineering. New Jersey: John Wiley \& Sons, 2011.

[48] J.-M. Redouté and M. Steyaert, EMC of Analog Integrated Circuits. Dordrecht: Springer Science \& Business Media, 2009.

[49] H. E. Stinehelfer, "An Accurate Calculation of Uniform Microstrip Transmission Lines," IEEE J. SolidState Circuits, vol. 3, no. 2, pp. 101-106, Jun. 1968.

[50] V. Teppati, A. Ferrero, and M. Sayed, Eds., Modern RF and Microwave Measurement Techniques. Cambridge: Cambridge University Press, 2013. 